\title{
EXACT RELATIONSHIPS BETWEEN CERTAIN CHARACTERISTICS OF GROWTH FOR COMPLEX SEQUENCES
}

\author{
G.G. BRAICHEV
}

\begin{abstract}
We establish exact estimates relating the classical densities of complex sequences (ordinary and averaged) with relative densities and lacunarity and sparsity indices.
\end{abstract}

Keywords: the upper and lower (average) densities, lacunarity and sparsity indices of sequence.

Mathematics Subject Classification: 11B05, 11L07

One of the main directions of the theory of entire functions is the studying the dependence of the function growth on the distribution of its zeroes on the plane. A large amount of the works related to this part of the complex analysis is devoted to the estimates of the indicators and types of an entire function of finite order via such usual characteristics of the behavior of its zeroes as usual averaged and other densities (see, for instance, [1]- [5] and the survey [6]). Recently also less traditional lacunarity and sparsity indices [7], [8] were begun being used. Recent studies of extremal problems for entire functions with zeroes on a ray [9], [10] clearly demonstrated the impossibility of obtaining final results without understanding internal connections between the densities of zeroes sequences.

As one can see at the example of work [8], the further development of the theory of extremal problems generates the need in finding and studying laws connecting "quantitative" density characteristics of tending a sequence to infinity with "qualitative" characteristics like lacunarity and sparsity indices. By the present such connections are not studied well enough. Covering this gap, here we find an exact connection of the aforementioned characteristics of growth for the zeroes sequence of entire functions of finite order.

Thus, the subject of the work are sequences of complex numbers $\Lambda=\left\{\lambda_{n}\right\}_{n=1}^{\infty}$ tending to infinity, which we take in order of ascending the moduli

$$
0<\left|\lambda_{1}\right|=\ldots=\left|\lambda_{n_{1}}\right|<\left|\lambda_{n_{1}+1}\right|=\ldots=\left|\lambda_{n_{2}}\right|<\left|\lambda_{n_{2}+1}\right|=\ldots=\left|\lambda_{n_{3}}\right|<\ldots
$$

The indices $n_{k}$ at which the moduli of the terms of the sequence have jumps are called central indices of sequence $\Lambda$.

We denote by $n_{\Lambda}(x)=\sum_{\left|\lambda_{n}\right| \leqslant x} 1$ the counting function of this sequence and $N_{\Lambda}(x)=\int_{0}^{x} \frac{n_{\Lambda}(t)}{t} d t$ stands for its averaged counting function.

The convergence index for sequence $\Lambda$ (see [1]) is calculated by the formulae

$$
\rho_{\Lambda}=\varlimsup_{x \rightarrow+\infty} \frac{\ln n_{\Lambda}(x)}{\ln x}=\varlimsup_{x \rightarrow+\infty} \frac{\ln N_{\Lambda}(x)}{\ln x} .
$$

G.G. BraicheV, EXACT RELATIONSHIPS BETWEEN CERTAIN CHARACTERISTICS OF GROWTH FOR COMPLEX SEQUENCES.

(C) Braichev G.G., 2013.

The work is supported by RFBR (grant 13-01-00281).

Submitted May 13, 2013. 
In what follows by $\rho$ we denote the convergence index $\rho_{\Lambda}$ for sequence $\Lambda$ assuming that $\rho$ is a positive number.

The quantities

$$
\bar{\Delta}_{\rho}(\Lambda)=\varlimsup_{x \rightarrow+\infty} \frac{n_{\Lambda}(x)}{x^{\rho}} \text { and } \bar{\Delta}_{\rho}^{*}(\Lambda)=\varlimsup_{x \rightarrow+\infty} \frac{N_{\Lambda}(x)}{x^{\rho}}
$$

are called upper $\rho$-densities (usual and averaged) of sequence $\Lambda$. Replacing the upper limits into the lower ones in these identities leads us to the definition of lower and averaged lower $\rho$-density of a sequence:

$$
\underline{\Delta}_{\rho}(\Lambda)=\varliminf_{x \rightarrow+\infty} \frac{n_{\Lambda}(x)}{x^{\rho}}, \quad \underline{\Delta}_{\rho}^{*}(\Lambda)=\varliminf_{x \rightarrow+\infty} \frac{N_{\Lambda}(x)}{x^{\rho}} .
$$

For the sake of simplification the notations, if it does not lead to an ambiguity, we shall omit the symbols $\rho$ and $\Lambda$ in the notations for densities and other introduced characteristics. Moreover, for the space saving, we shall use simultaneously both overline and underline assuming that they are in the correspondence in all the identities.

We introduce also upper and lower relative densities of a sequence assuming by the definition

$$
\underline{\bar{\nu}}=\varlimsup_{x \rightarrow+\infty} \frac{N(x)}{n(x)} .
$$

Since it always holds

$$
\overline{\bar{\Delta}}=\varlimsup_{x \rightarrow+\infty} \frac{n(x)}{x^{\rho}}=\varlimsup_{n \rightarrow \infty} \frac{n}{\left|\lambda_{n}\right|^{\rho}}=\varlimsup_{n \rightarrow \infty} \frac{n\left(\left|\lambda_{n}\right|\right)}{\left|\lambda_{n}\right|^{\rho}},
$$

then it is natural to introduce discrete averaged upper and lower density of a sequence by the formulae

$$
\widetilde{\Delta}=\varlimsup_{n \rightarrow \infty} \frac{N\left(\left|\lambda_{n}\right|\right)}{\left|\lambda_{n}\right|^{\rho}}
$$

We shall see later (see Theorem 1 and Proposition 1) that $\Delta^{*}=\Delta$, and if $\left|\lambda_{n}\right| \underset{n \rightarrow \infty}{\sim}\left|\lambda_{n+1}\right|$, then $\bar{\Delta}^{*}=\widetilde{\Delta}$.

The sequences satisfying condition $\bar{\Delta}^{*}=\Delta^{*}$, or, which is equivalent, $\bar{\Delta}=\Delta$, are called measurable. By analogy, as discrete measurable sequences we shall regard those satisfying the condition $\widetilde{\Delta}=\Delta$, meaning the existence of the limit $\lim _{n \rightarrow \infty} \frac{N\left(\left|\lambda_{n}\right|\right)}{\left|\lambda_{n}\right| \rho}$. In the same way, if there exists the limit $\lim _{x \rightarrow+\infty} \frac{N(x)}{n(x)}$, we shall say that such sequence is internal measurable. It is useful to note that in distinction to usual or discrete measurability, the notion of internal measurability is not associated with any index $\rho$.

We first of all note that none of discrete and internal measurability implies its measurability. It was shown in work [10] that the class of discrete measurable sequences is rather wide: for arbitrary numbers $\rho>0, \beta>0$ and $\alpha \in[0, \beta]$ there exist discrete measurable sequences with the densities $\Delta=\alpha$ and $\bar{\Delta}=\beta$. Here we prove (see below Proposition 9) that the same statement is valid also for internal measurable sequences.

By the definition of the counting function for sequence $\Lambda=\left\{\lambda_{n}\right\}$ we have $n_{\Lambda}(x)=0$ as $x \in\left[0,\left|\lambda_{1}\right|\right)$ and $n_{\Lambda}(x) \equiv n_{k}$ as $x \in\left[\left|\lambda_{n_{k}}\right|,\left|\lambda_{n_{k}+1}\right|\right), k=1,2, \ldots$ For the sequence of central indices $N=\left\{n_{k}\right\}_{k=1}^{\infty} \subseteq \mathbb{N}$ we define the characteristics

$$
\mu_{N}=\mu=\varlimsup_{k \rightarrow \infty} \frac{n_{k+1}}{n_{k}} \text { and } \delta_{N}=\delta=\varliminf_{k \rightarrow \infty} \frac{n_{k+1}}{n_{k}} .
$$

We shall call $\mu_{N}$ a lacunarity index, and $\delta_{N}$ will be called a sparsity index for sequence $N$. In the same we define lacunarity index $l_{\Lambda}$ and sparsity index $p_{\Lambda}$ for sequence $\Lambda=\left\{\lambda_{n}\right\} \subset \mathbb{C}$ (more 
precisely, for the sequence $\left.|\Lambda|=\left\{\left|\lambda_{n}\right|\right\} \subset \mathbb{R}_{+}\right)$:

$$
l_{\Lambda}=l=\varlimsup_{n \rightarrow \infty} \frac{\left|\lambda_{n+1}\right|}{\left|\lambda_{n}\right|}=\varlimsup_{k \rightarrow \infty} \frac{\left|\lambda_{n_{k+1}}\right|}{\left|\lambda_{n_{k}}\right|} \text { and } p_{\Lambda}=p=\varliminf_{k \rightarrow \infty} \frac{\left|\lambda_{n_{k+1}}\right|}{\left|\lambda_{n_{k}}\right|} .
$$

A sequence $\Lambda$ is called Hadamard lacunar if its lacunarity index is greater than one, i.e., $p_{\Lambda}>1$ (as $p_{\Lambda}=\infty$ we have so called Ostrowsky lacunas). If lacunarity index of sequence $\Lambda$ equals one, $l_{\Lambda}=1$, then $\Lambda$ is called weakly Hadamard lacunar (shortly weakly lacunar). For instance, the sequence of all prime numbers is weakly lacunar. As $b>1$, the same property is possessed by sequence $\Lambda=\left\{b^{n^{\alpha}}\right\}$ for $\alpha \in(0,1)$. If $\alpha=1$, then $p_{\Lambda}=l_{\Lambda}=b$, while for $\alpha>1$ it happens $p_{\Lambda}=\infty$.

The connections between usual and averaged densities of sequence $\Lambda$ are reflected by classical inequalities (see [1, Ch. I, Sec. 12], [2, Ch. II, Sec. 4, Subsec. 4]):

$$
\underline{\Delta} \leqslant \rho \underline{\Delta}^{*} \leqslant \rho \bar{\Delta}^{*} \leqslant \bar{\Delta} \leqslant \rho e \bar{\Delta}^{*} .
$$

In book [3, P. 16] the matter is an estimate specifying the latter inequality in the case when not only the upper but also the lower density of a sequence is known. Namely,

$$
\rho e \bar{\Delta}^{*} \geq \bar{\Delta} \exp \{\underline{\Delta} / \bar{\Delta}\} \text {. }
$$

The general results on a relative growth of convex functions established in monograph [7] imply the following inequalities improving all previous relations (see also [1]):

$$
\rho a_{1} \bar{\Delta}^{*} \leqslant \Delta \leqslant \rho \widetilde{a}_{1} \bar{\Delta}^{*}, \quad \rho \widetilde{a}_{2} \bar{\Delta}^{*} \leqslant \bar{\Delta} \leqslant \rho a_{2} \bar{\Delta}^{*} .
$$

Here $a_{1}$ and $a_{2}$ are the roots to equation

$$
a \ln \frac{e}{a}=\underline{\Delta}^{*} / \bar{\Delta}^{*}
$$

while $\widetilde{a}_{1}$ and $\widetilde{a}_{2}$ are the roots of a similar equation with a "corrected" right hand side

$$
a \ln \frac{e}{a}=\widetilde{\Delta} / \bar{\Delta}^{*}
$$

The roots to these equations are related by the inequalities $0 \leqslant a_{1} \leqslant \widetilde{a}_{1} \leqslant 1 \leqslant \widetilde{a}_{2} \leqslant a_{2} \leqslant e$.

In view of $(2)$, by the condition $\widetilde{\Delta}=\underline{\Delta}^{*}$ (see Theorem 1 below) for discrete measurable sequences the exact identities

$$
\underline{\Delta}=\rho a_{1} \bar{\Delta}^{*}, \quad \bar{\Delta}=\rho a_{2} \bar{\Delta}^{*}
$$

hold true.

Thus, we can state that at the present the relations between usual and averaged densities of sequences are quite well studied. Unfortunately, this is not true for the relations between the densities (usual, averaged, relative) and the lacunarity and sparsity indices of sequence $\Lambda$. At the same time, such study is of great value in studying the growth of entire functions whose zeroes are $\Lambda$.

The present work is devoted to finding out the exact relations between the mentioned characteristics of sequences.

In what follows we assume that number $\rho>0$ is given and the corresponding quantities are calculated for this index $\rho$. Moreover, it follows from inequalities (1) that the conditions $\bar{\Delta}=0$ and $\bar{\Delta}^{*}=0$, as well as conditions $\bar{\Delta}=\infty$ and $\bar{\Delta}^{*}=\infty$ are equivalent. This is why in what follows we assume and sometimes we say it explicitly that the condition $0<\bar{\Delta}<\infty$ is satisfied. 
Proposition 1. The inequalities

$$
\begin{gathered}
\Delta \mu \leqslant \bar{\Delta}, \\
\underline{\Delta} l^{\rho} \leqslant \bar{\Delta}, \\
\bar{\Delta}^{*} \leqslant \widetilde{\Delta} l^{\rho}, \\
\underline{\nu} \mu \leqslant \bar{\nu}, \\
\ln l \leqslant \bar{\nu}-\underline{\nu} .
\end{gathered}
$$

Proof. Since the counting function of sequence $\Lambda$ satisfy the identities $n_{\Lambda}(t)=n(t) \equiv n_{k}$ as $t \in I_{k}=\left[\left|\lambda_{n_{k}}\right|,\left|\lambda_{n_{k}+1}\right|\right), k=1,2, \ldots$,

$$
\begin{gathered}
\bar{\Delta}=\varlimsup_{k \rightarrow \infty} \sup _{x \in I_{k}} \frac{n(x)}{x^{\rho}}=\varlimsup_{k \rightarrow \infty} \frac{n_{k}}{\left|\lambda_{n_{k}}\right|^{\rho}}, \quad \Delta=\varliminf_{k \rightarrow \infty} \inf _{x \in I_{k}} \frac{n(x)}{x^{\rho}}=\underline{\lim _{k \rightarrow \infty}} \frac{n_{k}}{\left|\lambda_{n_{k+1}}\right|^{\rho}} \\
\bar{\nu}=\varlimsup_{k \rightarrow \infty} \sup _{x \in I_{k}} \frac{N(x)}{n(x)}=\varlimsup_{k \rightarrow \infty} \frac{N\left(\left|\lambda_{n_{k+1}}\right|\right)}{n_{k}}, \quad \underline{\nu}=\varliminf_{k \rightarrow \infty} \inf _{x \in I_{k}} \frac{N(x)}{n(x)}=\varliminf_{k \rightarrow \infty} \frac{N\left(\left|\lambda_{n_{k}}\right|\right)}{n_{k}} .
\end{gathered}
$$

By (9) we easily get

$$
\bar{\Delta}=\varlimsup_{k \rightarrow \infty} \frac{n_{k}}{\left|\lambda_{n_{k+1}}\right|^{\rho}} \frac{n_{k+1}}{n_{k}} \geq \Delta \varlimsup_{k \rightarrow \infty} \frac{n_{k+1}}{n_{k}}=\Delta \mu
$$

i.e., inequality (4) holds true. In the same way

$$
\bar{\Delta}=\varlimsup_{k \rightarrow \infty} \frac{n_{k}}{\left|\lambda_{n_{k+1}}\right|^{\rho}} \frac{\left|\lambda_{n_{k+1}}\right|^{\rho}}{\left|\lambda_{n_{k}}\right|^{\rho}} \geq \triangleq \varlimsup_{k \rightarrow \infty} \frac{\left|\lambda_{n_{k+1}}\right|^{\rho}}{\left|\lambda_{n_{k}}\right|^{\rho}}=\Delta l^{\rho},
$$

that justifies the validity of inequality (5).

In the same way by (10) we obtain (7):

$$
\bar{\nu}=\varlimsup_{k \rightarrow \infty} \frac{N\left(\left|\lambda_{n_{k+1}}\right|\right)}{n_{k}} \geq \varliminf_{k \rightarrow \infty} \frac{N\left(\left|\lambda_{n_{k+1}}\right|\right)}{n_{k+1}} \varlimsup_{k \rightarrow \infty} \frac{n_{k+1}}{n_{k}}=\underline{\nu} \mu .
$$

Due to the increasing of function $N(x)$, for arbitrary $\varepsilon>0$ and sufficiently large $k$ for each $x \in I_{k}$ we have

$$
\frac{N(x)}{x^{\rho}} \leqslant \frac{N\left(\left|\lambda_{n_{k+1}}\right|\right)}{\left|\lambda_{n_{k}}\right|^{\rho}}=\frac{N\left(\left|\lambda_{n_{k+1}}\right|\right)}{\left|\lambda_{n_{k+1}}\right|^{\rho}}\left(\frac{\left|\lambda_{n_{k+1}}\right|}{\left|\lambda_{n_{k}}\right|}\right)^{\rho}<(\widetilde{\Delta}+\varepsilon)(l+\epsilon)^{\rho} .
$$

Passing to the upper limit as $x \rightarrow+\infty$, and then as $\varepsilon \rightarrow 0$, we prove (6).

To prove (8) and in what follows we shall make use of the following formula

$$
N\left(\left|\lambda_{n_{k+1}}\right|\right)-N\left(\left|\lambda_{n_{k}}\right|\right)=n_{k} \ln \frac{\left|\lambda_{n_{k+1}}\right|}{\left|\lambda_{n_{k}}\right|},
$$

which follows directly from the definition of function $N(x)$ :

$$
N\left(\left|\lambda_{n_{k+1}}\right|\right)-N\left(\left|\lambda_{n_{k}}\right|\right)=\int_{\left|\lambda_{n_{k}}\right|}^{\left|\lambda_{n_{k+1}}\right|} \frac{n(x)}{x} d x=n_{k} \int_{\left|\lambda_{n_{k}}\right|}^{\left|\lambda_{n_{k+1}}\right|} \frac{d x}{x}=n_{k} \ln \frac{\left|\lambda_{n_{k+1}}\right|}{\left|\lambda_{n_{k}}\right|} .
$$

To obtain (8), we write (11) in a convenient form

$$
\ln \frac{\left|\lambda_{n_{k+1}}\right|}{\left|\lambda_{n_{k}}\right|}=\frac{N\left(\left|\lambda_{n_{k+1}}\right|\right)}{n_{k}}-\frac{N\left(\left|\lambda_{n_{k}}\right|\right)}{n_{k}}
$$

and pass to the upper limit taking into account formulae (10). The proof is complete.

The proven proposition implies the following fact. 
Corollary. If a sequence is measurable $(\underline{\Delta}=\bar{\Delta}=\Delta)$ with $\Delta>0$ or only internal measurable $(\underline{\nu}=\bar{\nu}=\nu)$ with $\nu>0$, then this sequence and the sequence of its central indices are weakly lacunar.

Indeed, the first statement is implied by formulae (4) and (5), while the other follows from inequalities (7) and (8).

The next proposition states relations between usual, relative and discrete densities of sequences.

Proposition 2. The inequalities

$$
\underset{\sim}{\Delta} \leqslant \min \{\underline{\Delta} \bar{\nu}, \bar{\Delta} \underline{\nu}\} \leqslant \max \{\underline{\Delta} \bar{\nu}, \bar{\Delta} \underline{\nu}\} \leqslant \widetilde{\Delta}
$$

hold true.

Proof. Employing formulae (9) and (10), we get

or

$$
\Delta=\varliminf_{k \rightarrow \infty} \frac{n_{k}}{\left|\lambda_{n_{k+1}}\right| \rho} \frac{N\left(\left|\lambda_{n_{k+1}}\right|\right)}{n_{k}} \leqslant \varliminf_{k \rightarrow \infty} \frac{n_{k}}{\left|\lambda_{n_{k+1}}\right| \rho} \varlimsup_{k \rightarrow \infty} \frac{N\left(\left|\lambda_{n_{k+1}}\right|\right)}{n_{k}}=\Delta \bar{\nu}
$$

$$
\Delta=\varliminf_{k \rightarrow \infty} \frac{n_{k}}{\left|\lambda_{n_{k}}\right|^{\rho}} \frac{N\left(\left|\lambda_{n_{k}}\right|\right)}{n_{k}} \leqslant \varlimsup_{k \rightarrow \infty} \frac{n_{k}}{\left|\lambda_{n_{k}}\right|^{\rho}} \varliminf_{k \rightarrow \infty} \frac{N\left(\left|\lambda_{n_{k}}\right|\right)}{n_{k}}=\bar{\Delta} \underline{\nu} .
$$

Thus, the inequality $\underset{\sim}{\Delta} \leqslant \min \{\underline{\Delta} \bar{\nu}, \bar{\Delta} \underline{\nu}\}$ is proven.

The inequality $\max \{\underline{\Delta} \bar{\nu}, \bar{\Delta} \underline{\nu}\} \leqslant \widetilde{\Delta}$ is checked in the same way

$$
\begin{gathered}
\widetilde{\Delta}=\varlimsup_{k \rightarrow \infty} \frac{n_{k}}{\left|\lambda_{n_{k+1}}\right|^{\rho}} \frac{N\left(\left|\lambda_{n_{k+1}}\right|\right)}{n_{k}} \geq \varliminf_{k \rightarrow \infty} \frac{n_{k}}{\left|\lambda_{n_{k+1}}\right|^{\rho}} \varlimsup_{k \rightarrow \infty} \frac{N\left(\left|\lambda_{n_{k+1}}\right|\right)}{n_{k}}=\Delta \bar{\nu}, \\
\widetilde{\Delta}=\varlimsup_{k \rightarrow \infty} \frac{n_{k}}{\left|\lambda_{n_{k}}\right|^{\rho}} \frac{N\left(\left|\lambda_{n_{k}}\right|\right)}{n_{k}} \geq \varlimsup_{k \rightarrow \infty} \frac{n_{k}}{\left|\lambda_{n_{k}}\right| \rho} \varliminf_{k \rightarrow \infty} \frac{N\left(\left|\lambda_{n_{k}}\right|\right)}{n_{k}}=\bar{\Delta} \underline{\nu} .
\end{gathered}
$$

The proof is complete.

In book [12, Part IV, Ch. 1, Problem 60]) the connection between relative densities of a sequence and its convergence index was indicated

$$
\underline{\nu} \leqslant 1 / \rho \leqslant \bar{\nu} .
$$

Which properties does a sequence possesses once the identity is realized in one of these inequalities? In our opinion, Proposition 2 can provide an interesting information on this issue.

Corollary. If the upper relative density of a sequence attains its minimum, i.e., $\bar{\nu}=1 / \rho$, then the identities $\underline{\Delta}^{*}=\Delta=\Delta / \rho$ hold true. If the lower relative density of a sequence attains its maximum, i.e., $\underline{\nu}=1 / \rho$, then $\bar{\Delta}^{*}=\widetilde{\Delta}=\bar{\Delta} / \rho$.

Indeed, it follows from (1) that $\Delta / \rho \leqslant \underline{\Delta}^{*}$. Under the condition $\bar{\nu}=1 / \rho$ the left estimate in (13) yields $\underset{\sim}{\Delta} \leqslant / \rho$. Hence,

$$
\underline{\Delta} / \rho \leqslant \underline{\Delta}^{*} \leqslant \Delta \leqslant \Delta / \rho
$$

that leads us to the first statement of the corollary. The second follows from similar arguments leading us to the inequalities

$$
\bar{\Delta} / \rho \leqslant \widetilde{\Delta} \leqslant \bar{\Delta}^{*} \leqslant \bar{\Delta} / \rho
$$

in the case $\underline{\nu}=1 / \rho$. 
Proposition 3. For each sequence of complex numbers with the convergence index $\rho>0$ the following statements hold true.

If $0<\bar{\Delta}<\infty$, then the inequality

$$
l^{\rho} \geq \delta
$$

is valid and if $0<\Delta<\infty$, we have

$$
p^{\rho} \leqslant \mu
$$

The estimates

$$
\begin{aligned}
& \ln p \leqslant \underline{\nu}(\mu-1), \\
& \ln l \geq \bar{\nu} \frac{\delta-1}{\delta}
\end{aligned}
$$

hold true.

Proof. Under the condition $\bar{\Delta} \neq 0$ we can write

$$
\bar{\Delta}=\varlimsup_{k \rightarrow \infty} \frac{n_{k}}{\left|\lambda_{n_{k}}\right|^{\rho}} \leqslant \varlimsup_{k \rightarrow \infty} \frac{n_{k+1}}{\left|\lambda_{n_{k+1}}\right|^{\rho}} \frac{1}{\underline{\lim _{k \rightarrow \infty}} \frac{n_{k+1}}{n_{k}}} \varlimsup_{k \rightarrow \infty}\left(\frac{\left|\lambda_{n_{k+1}}\right|}{\left|\lambda_{n_{k}}\right|}\right)^{\rho}=\bar{\Delta} \frac{l^{\rho}}{\delta} .
$$

Canceling out by $\bar{\Delta}$, we obtain inequality (14). If $\underline{\Delta} \neq 0$, then

$$
\underline{\Delta}=\varliminf_{k \rightarrow \infty} \frac{n_{k}}{\left|\lambda_{n_{k+1}}\right|^{\rho}} \leqslant \varliminf_{k \rightarrow \infty} \frac{n_{k+1}}{\left|\lambda_{n_{k+2}}\right|^{\rho}} \frac{1}{\varlimsup_{k \rightarrow \infty} \frac{n_{k+1}}{n_{k}}} \varliminf_{k \rightarrow \infty}\left(\frac{\left|\lambda_{n_{k+2}}\right|}{\left|\lambda_{n_{k+1}}\right|}\right)^{\rho}=\triangleq \frac{p^{\rho}}{\mu}
$$

that implies inequality (15).

Estimates (16), (17) can be proven by a generalized Stolz-Cesáro theorem (see, for instance, [7]) and formulae (12), (10):

$$
\begin{aligned}
\ln p & =\varliminf_{k \rightarrow \infty} \ln \frac{\left|\lambda_{n_{k+1}}\right|}{\left|\lambda_{n_{k}}\right|}=\varliminf_{k \rightarrow \infty} \frac{N\left(\left|\lambda_{n_{k+1}}\right|\right)-N\left(\left|\lambda_{n_{k}}\right|\right)}{n_{k}} \\
& =\varliminf_{k \rightarrow \infty} \frac{N\left(\left|\lambda_{n_{k+1}}\right|\right)-N\left(\left|\lambda_{n_{k}}\right|\right)}{n_{k+1}-n_{k}}\left(\frac{n_{k+1}}{n_{k}}-1\right) \\
& \leqslant \varliminf_{k \rightarrow \infty} \frac{N\left(\left|\lambda_{n_{k+1}}\right|\right)-N\left(\left|\lambda_{n_{k}}\right|\right)}{n_{k+1}-n_{k}} \varlimsup_{k \rightarrow \infty}\left(\frac{n_{k+1}}{n_{k}}-1\right) \\
& \leqslant \varliminf_{k \rightarrow \infty} \frac{N\left(\left|\lambda_{n_{k}}\right|\right)}{n_{k}}(\mu-1)=\underline{\nu}(\mu-1) .
\end{aligned}
$$

Arguing in the same way, we obtain

$$
\begin{aligned}
\ln l & =\varlimsup_{k \rightarrow \infty} \ln \frac{\left|\lambda_{n_{k+1}}\right|}{\left|\lambda_{n_{k}}\right|}=\varlimsup_{k \rightarrow \infty} \frac{N\left(\left|\lambda_{n_{k+1}}\right|\right)-N\left(\left|\lambda_{n_{k}}\right|\right)}{n_{k}} \\
& =\varlimsup_{k \rightarrow \infty} \frac{N\left(\left|\lambda_{n_{k+1}}\right|\right)-N\left(\left|\lambda_{n_{k}}\right|\right)}{n_{k}-n_{k-1}}\left(\frac{n_{k}-n_{k-1}}{n_{k}}\right) \\
& \geq \varlimsup_{k \rightarrow \infty} \frac{N\left(\left|\lambda_{n_{k+1}}\right|\right)-N\left(\left|\lambda_{n_{k}}\right|\right)}{n_{k}-n_{k-1}} \varliminf_{k \rightarrow \infty}\left(1-\frac{1}{n_{k} / n_{k-1}}\right) \geq \varlimsup_{k \rightarrow \infty} \frac{N\left(\left|\lambda_{n_{k}}\right|\right)}{n_{k}}\left(1-\frac{1}{\delta}\right),
\end{aligned}
$$

i.e., inequality $\ln l \geq \bar{\nu} \frac{\delta-1}{\delta}$. The proof is complete.

We shall also need several simply checked facts and it is convenient to summarize them in the following statement. 
Lemma. I. The function

$$
q_{1}(x)=\left\{\begin{array}{cc}
\frac{\ln x}{x-1}, & x \neq 1 \\
1, & x=1
\end{array}\right.
$$

strictly decreases on $(0,+\infty)$.

II. The function $q_{2}(x)=\frac{e^{x-1}}{x}$ has the unique minimum in $(0,+\infty)$; this minimum equals 1 and is attained at the point $x=1$.

III. The function $q_{3}(x)=\frac{b+a \ln x}{x^{\rho}}, a>0, b>0$ has the unique maximum in $(0,+\infty)$; this maximum equals $\frac{a}{\rho} e^{\rho \frac{b}{a}-1}$ and it attained at the point $x=e^{\frac{1}{\rho}-\frac{b}{a}}$.

Let us study now the dependence between the sparsity index and its usual and discrete densities.

Proposition 4. Sparsity index, the density and discrete densities of a sequence with convergence index $\rho>0$ are related by the inequalities

$$
\left(p^{\rho}-1\right) \widetilde{\Delta} \leqslant \bar{\Delta} \ln p, \quad \underline{\Delta} p^{\rho} \ln p \leqslant\left(p^{\rho}-1\right) \underset{\sim}{\Delta} .
$$

Proof. As $p=1$, both the inequalities become to a trivial identity $0=0$. As $p>1$, we denote $c_{k}=\frac{\left|\lambda_{n_{k+1}}\right|}{\left|\lambda_{n_{k}}\right|}$ and apply Stolz-Cesáro theorem employing identity (10) and Item I of the previous lemma:

$$
\begin{aligned}
\widetilde{\Delta} & =\varlimsup_{k \rightarrow \infty} \frac{N\left(\left|\lambda_{n_{k}}\right|\right)}{\left|\lambda_{n_{k}}\right|^{\rho}} \leqslant \varlimsup_{k \rightarrow \infty} \frac{N\left(\left|\lambda_{n_{k+1}}\right|\right)-N\left(\left|\lambda_{n_{k}}\right|\right)}{\left|\lambda_{n_{k+1}}\right|^{\rho}-\left|\lambda_{n_{k}}\right|^{\rho}}=\varlimsup_{k \rightarrow \infty} \frac{n_{k}}{\left|\lambda_{n_{k}}\right|^{\rho}} \frac{\ln c_{k}}{c_{k}^{\rho}-1} \\
& \leqslant \frac{1}{\rho} \varlimsup_{k \rightarrow \infty} \frac{n_{k}}{\left|\lambda_{n_{k}}\right|^{\rho}} \varlimsup_{k \rightarrow \infty} \frac{\ln c_{k}^{\rho}}{c_{k}^{\rho}-1}=\frac{\bar{\Delta}}{\rho} \cdot \frac{\ln p^{\rho}}{p^{\rho}-1} .
\end{aligned}
$$

Thus, $\widetilde{\Delta} \leqslant \bar{\Delta} \cdot \frac{\ln p}{p^{\rho}-1}$. In the same way,

$$
\begin{aligned}
\underset{\sim}{\Delta} & =\varliminf_{k \rightarrow \infty} \frac{N\left(\left|\lambda_{n_{k}}\right|\right)}{\left|\lambda_{n_{k}}\right|^{\rho}} \geq \varliminf_{k \rightarrow \infty} \frac{N\left(\left|\lambda_{n_{k+1}}\right|\right)-N\left(\left|\lambda_{n_{k}}\right|\right)}{\left|\lambda_{n_{k+1}}\right|^{\rho}-\left|\lambda_{n_{k}}\right|^{\rho}}=\underline{\lim _{k \rightarrow \infty}} \frac{n_{k}}{\left|\lambda_{n_{k+1}}\right|^{\rho}} \frac{\ln c_{k}}{1-c_{k}^{-\rho}} \\
& \geq \frac{1}{\rho} \varliminf_{k \rightarrow \infty} \frac{n_{k}}{\left|\lambda_{n_{k+1}}\right| \rho} \varliminf_{k \rightarrow \infty} \frac{\ln c_{k}^{-\rho}}{c_{k}^{-\rho}-1}=\frac{\triangleq}{\rho} \cdot \frac{p^{\rho} \ln p^{\rho}}{p^{\rho}-1}
\end{aligned}
$$

i.e., $\underset{\sim}{\Delta} \geq \Delta \cdot \frac{p^{\rho} \ln p}{p^{\rho}-1}$. The proof is complete.

As a corollary of Proposition 2-4 we can obtain

Proposition 5. If $0<\bar{\Delta}<\infty$ and the sequence $\left\{\lambda_{n_{k}}\right\}_{k=1}^{\infty}$ has Hadamard lacunas, then the estimates

$$
\frac{\ln p}{\mu-1} \leqslant \underline{\nu} \leqslant \frac{\ln p}{p^{\rho}-1}
$$

hold true.

If $0<\Delta<\infty$ and the sequence $\left\{n_{k}\right\}_{k=1}^{\infty}$ has Hadamard lacunas, then the estimates

$$
\frac{p^{\rho} \ln p}{p^{\rho}-1} \leqslant \bar{\nu} \leqslant \frac{\delta \ln l}{\delta-1}
$$

are valid. 
Proof. Since $\left\{\lambda_{n_{k}}\right\}_{k=1}^{\infty}$ has Hadamard lacunas, we have $p>1$. Thus, comparing the right hand side of inequalities (13) with the first inequality in formula (18), we obtain $\underline{\nu} \bar{\Delta} \leqslant \widetilde{\Delta} \leqslant \bar{\Delta} \frac{\ln p}{p^{\rho}-1}$. It implies the right hand side of relation (19). The left hand side of this relations follows from (16), since in accordance with (15) we have $\mu \geq p^{\rho}>1$.

In the proof of estimates (20) we act in the same way, now we just combine the left hand side of (13) and the second inequality in (18) and apply then (17). The proof is complete.

The result is exact in the following sense. The identities in (19), (20) hold if the same is true for (14), (15). And it holds, for instance, for each sequence $\Lambda=\left\{\lambda_{n}\right\}_{n=1}^{\infty}$ such that the moduli of its terms and its central indices form two coherent "almost geometric" sequences, i.e., for some $q>1$ the satisfy the conditions

$$
\left|\lambda_{n_{k+1}}\right| \sim q\left|\lambda_{n_{k}}\right|, \quad n_{k+1} \sim q^{\rho} n_{k}, \quad k \rightarrow \infty .
$$

In this case $l=p=q, \mu=\delta=q^{\rho}$ and the formulae

$$
\underline{\nu}=\frac{\ln q}{q^{\rho}-1}, \quad \bar{\nu}=\frac{q^{\rho} \ln q}{q^{\rho}-1}
$$

are valid.

Theorem 1. The lower discrete averaged density $\underset{\sim}{\Delta}$ and lower averaged density $\Delta^{*}$ an arbitrary sequence $\Lambda \subset \mathbb{C}$ tending to infinity coincide

$$
\Delta=\Delta^{*}
$$

Proof. It is obvious that

$$
\underset{\sim}{\Delta} \varliminf_{n \rightarrow \infty} \frac{N\left(\left|\lambda_{n}\right|\right)}{\left|\lambda_{n}\right|^{\rho}} \geq \varliminf_{x \rightarrow+\infty} \frac{N(x)}{x^{\rho}}=\underline{\Delta}^{*}
$$

In order to obtain the inverse inequality, we consider function $\Phi(x)=\frac{N(x)}{x^{\rho}}$ and to study it for $k \in \mathbb{N}$ we let

$$
\Phi_{k}(t)=\frac{N\left(\left|\lambda_{n_{k}}\right|\right)+n_{k} \ln t}{t^{\rho}}, \quad t>0
$$

Since on the intervals $I_{k}=\left[\left|\lambda_{n_{k}}\right|,\left|\lambda_{n_{k}+1}\right|\right)$ we have $n_{\Lambda}(t) \equiv n_{k}$, then

$$
\Phi(x)=\frac{N\left(\left|\lambda_{n_{k}}\right|\right)+n_{k} \ln \frac{x}{\left|\lambda_{n_{k}}\right|}}{x^{\rho}}=\frac{1}{\left|\lambda_{n_{k}}\right| \rho} \Phi_{k}\left(\frac{x}{\left|\lambda_{n_{k}}\right|}\right), \quad x \in I_{k} .
$$

According to Item III of Lemma, $\Phi(x)$ is either monotone on $I_{k}$ or it has the unique maximum on this interval. In any case we have

$$
\inf _{x \in I_{k}}\{\Phi(x)\}=\min \left\{\Phi\left(\left|\lambda_{n_{k}}\right|\right), \Phi\left(\left|\lambda_{n_{k+1}}\right|\right)\right\}, \quad k \in \mathbb{N} .
$$

Hence,

$$
\begin{aligned}
\underline{\Delta}^{*} & =\varliminf_{k \rightarrow \infty} \inf _{x \in I_{k}} \Phi(x)=\varliminf_{k \rightarrow \infty} \min \left\{\Phi\left(\left|\lambda_{n_{k}}\right|\right), \Phi\left(\left|\lambda_{n_{k+1}}\right|\right)\right\} \\
& \geq \varliminf_{k \rightarrow \infty} \operatorname{linf}_{m \geq k} \Phi\left(\left|\lambda_{m}\right|\right)=\varliminf_{m \rightarrow \infty} \Phi\left(\left|\lambda_{m}\right|\right)=\underset{\sim}{\Delta}, \text { i.e. } \quad \underline{\lim }^{*} \geq \underset{\sim}{\Delta} .
\end{aligned}
$$

Comparing with the previous relation, we obtain $\underset{\sim}{\Delta}=\underline{\Delta}^{*}$. The proof is complete.

In the formulation of the next result we make use of the following standard notation $a^{+}=$ $\max \{a, 0\}$. 
Theorem 2. For each sequence $\Lambda \subset \mathbb{C}$ tending to infinity with the averaged upper $\rho$-density $\bar{\Delta}_{\rho}^{*}(\Lambda)=\bar{\Delta}^{*} \in(0,+\infty)$ the inequalities

$$
\begin{aligned}
& \bar{\Delta}^{*} \leqslant \widetilde{\Delta} \frac{e^{\rho \underline{\nu}-1}}{\rho \underline{\nu}}, \\
& \bar{\Delta}^{*} \leqslant \widetilde{\Delta} \frac{e^{\rho \bar{\nu}-1}}{\rho \bar{\nu}} \\
& \bar{\Delta}^{*} \leqslant \widetilde{\Delta} \frac{l^{\rho}}{\ln l^{\rho}+1}, \\
& \bar{\Delta}^{*} \leqslant \widetilde{\Delta} \frac{l^{-\rho}}{\left(\ln l^{-\rho}+1\right)^{+}}
\end{aligned}
$$

hold true.

Proof. First of all we observe that inequality (23) specifies inequality (5) in the case $l>1$. Now we proceed to the proof.

If $\widetilde{\Delta}=\bar{\Delta}^{*}$, inequalities (21)-(24) are obviously true since each factor in quantity $\widetilde{\Delta}$ in the right hand sides of these inequalities is at least one.

Let us study the case $\widetilde{\Delta}<\bar{\Delta}^{*}$. We choose a positive number $\varepsilon<\bar{\Delta}^{*}-\widetilde{\Delta}$. Keeping the notations of Theorem 1 and employing the definition of upper averaged density, we find the sequences of indices $\mathbb{K}$ and points $x_{k}$ so that the relations

$$
\begin{aligned}
& \bar{\Delta}^{*}=\varlimsup_{k \rightarrow \infty} \sup _{x \in I_{k}} \Phi(x)=\lim _{k \in \mathbb{K}} \sup _{x \in I_{k}} \Phi(x), \\
& \Phi\left(x_{k}\right)=\sup _{x \in I_{k}} \Phi(x)>\widetilde{\Delta}+\varepsilon, \quad k \in \mathbb{K}
\end{aligned}
$$

hold true. For sufficiently great indices $k$, at the end-points of segment $I_{k}$ function $\Phi(x)$ takes the values

$$
\Phi\left(\left|\lambda_{n_{k}}\right|\right)<\widetilde{\Delta}+\varepsilon<\Phi\left(x_{k}\right)
$$

Thus, neglecting a finite number of the indices in $\mathbb{K}$ if it is needed, we can suppose for each $k \in \mathbb{K}$ the point $x_{k}$ lies strictly inside $I_{k}$, i.e.,

$$
\left|\lambda_{n_{k}}\right|<x_{k}<\left|\lambda_{n_{k+1}}\right|, \quad k \in \mathbb{K}
$$

We denote $c_{k}=\frac{\left|\lambda_{n_{k+1}}\right|}{\left|\lambda_{n_{k}}\right|}$ and $\nu_{k}=\frac{N\left(\left|\lambda_{n_{k}}\right|\right)}{n_{k}}$. Applying Item III of Lemma to function $\Phi_{k}(t)$, due to the identity

we obtain

$$
\Phi(x)=\frac{1}{\left|\lambda_{n_{k}}\right|^{\rho}} \Phi_{k}\left(\frac{x}{\left|\lambda_{n_{k}}\right|}\right), \quad x \in I_{k}
$$

$$
\begin{gathered}
1<\frac{x_{k}}{\left|\lambda_{n_{k}}\right|}=e^{\frac{1}{\rho}-\nu_{k}}<c_{k}, \\
\Phi\left(x_{k}\right)=\frac{n_{k}}{\left|\lambda_{n_{k}}\right|^{\rho}} e^{\rho \nu_{k}-1}=\frac{N\left(\left|\lambda_{n_{k}}\right|\right)}{\left|\lambda_{n_{k}}\right| \rho} \frac{e^{\rho \nu_{k}-1}}{\rho \nu_{k}}=\Phi\left(\left|\lambda_{n_{k}}\right|\right) \frac{e^{\rho \nu_{k}-1}}{\rho \nu_{k}} .
\end{gathered}
$$

Finding the logarithm of (25), we arrive at the inequality

$$
1-\ln c_{k}^{\rho}<\rho \nu_{k}<1 .
$$

After the above preliminary work, estimate (21) can be easily proven by passing to a limit in (26):

$$
\bar{\Delta}^{*}=\lim _{k \in \mathbb{K}} \Phi\left(x_{k}\right)=\lim _{k \in \mathbb{K}}\left\{\Phi\left(\left|\lambda_{n_{k}}\right|\right) \frac{e^{\rho \nu_{k}-1}}{\rho \nu_{k}}\right\} \leqslant \varlimsup_{k \rightarrow \infty} \Phi\left(\left|\lambda_{n_{k}}\right|\right) \varlimsup_{k \rightarrow \infty} \frac{e^{\rho \nu_{k}-1}}{\rho \nu_{k}} \leqslant \widetilde{\Delta} \frac{e^{\rho \underline{\nu}-1}}{\rho \underline{\nu}} .
$$


Here we have used that $q_{2}(x)=\frac{e^{x-1}}{x}$ decreases on the interval $(0,1)$ in accordance with Item II of Lemma.

Inequality (22) can be proven in the same way. We write

$$
\Phi(x)=\frac{N\left(\left|\lambda_{n_{k+1}}\right|\right)+n_{k} \ln \frac{x}{\left|\lambda_{n_{k+1}}\right|}}{x^{\rho}}=\frac{1}{\left|\lambda_{n_{k+1}}\right|^{\rho}} \Phi_{k}\left(\frac{x}{\left|\lambda_{n_{k+1}}\right|}\right), \quad x \in I_{k} .
$$

We denote $\nu_{k}^{\prime}=\frac{N\left(\left|\lambda_{n_{k+1}}\right|\right)}{n_{k}}$ to obtain

$$
\frac{1}{c_{k}}<\frac{x}{\left|\lambda_{n_{k+1}}\right|}=e^{\frac{1}{\rho}-\nu_{k}^{\prime}}<1
$$

And finding the logarithm,

$$
1<\rho \nu_{k}^{\prime}<1+\ln c_{k}^{\rho} .
$$

The next relation is deduced completely in the same way as formula (25) and it casts into the form

$$
\Phi\left(x_{k}\right)=\frac{n_{k}}{\left|\lambda_{n_{k+1}}\right|^{\rho}} e^{\rho \nu_{k}^{\prime}-1}=\frac{N\left(\left|\lambda_{n_{k+1}}\right|\right)}{\left|\lambda_{n_{k+1}}\right| \rho} \frac{e^{\rho \nu_{k}^{\prime}-1}}{\rho \nu_{k}^{\prime}}=\Phi\left(\left|\lambda_{n_{k+1}}\right|\right) \frac{e^{\rho \nu_{k}^{\prime}-1}}{\rho \nu_{k}^{\prime}} .
$$

Using again Item II of Lemma, this time on interval $(1,+\infty)$, where function $q_{2}(x)$ increases, by the passage to a limit, from (28) we get (22):

$$
\bar{\Delta}^{*}=\lim _{k \in \mathbb{K}} \Phi\left(x_{k}\right) \leqslant \varlimsup_{k \rightarrow \infty} \Phi\left(\left|\lambda_{n_{k+1}}\right|\right) \varlimsup_{k \rightarrow \infty} \frac{e^{\rho \nu_{k}^{\prime}-1}}{\rho \nu_{k}^{\prime}} \leqslant \widetilde{\Delta} \frac{e^{\rho \bar{\nu}-1}}{\rho \bar{\nu}} .
$$

In order to prove inequality (23) we employ the definition of lacunarity index. According to this definition, for each $\varepsilon>0$ and each $k \geq k_{0}(\varepsilon)$ we have $c_{k}<l+\varepsilon$. For sufficiently large $k \in \mathbb{K}$, it allows us to obtain by (28) that $1<\rho \nu_{k}^{\prime}<1+\ln (l+\epsilon)^{\rho}$, and then by (29) and Item II we get

$$
\Phi\left(x_{k}\right)<\Phi\left(\left|\lambda_{n_{k+1}}\right|\right) \frac{e^{\ln (l+\varepsilon)^{\rho}}}{\ln (l+\varepsilon)+1}<(\widetilde{\Delta}+\varepsilon) \frac{(l+\varepsilon)^{\rho}}{\ln (l+\varepsilon)+1} .
$$

Passing to the limits as $k \rightarrow \infty, k \in \mathbb{K}$ and $\varepsilon \rightarrow 0$, we arrive at the desired estimate (23).

The proof of inequality (24) follows similar lines. Indeed, the inequalities

$$
1-\ln (l+\epsilon)^{\rho}<1-\ln c_{k}<\rho \nu_{k}<1, \quad \rho \nu_{k}>0,
$$

follow from (27) that implies $\rho \nu_{k}>\left(1+\ln (l+\epsilon)^{-\rho}\right)^{+}$. The rest is clear. The proof is complete.

We note that estimates (21) and (22) of Theorem 2 can be written as

$$
\frac{\widetilde{\Delta}}{\bar{\Delta}^{*}} \geq \rho \underline{\nu} e^{1-\rho \underline{\nu}}=e^{1-\rho \underline{\nu}} \ln \frac{e}{e^{1-\rho \underline{\nu}}}, \quad \frac{\widetilde{\Delta}}{\overline{\bar{\Delta}}^{*}} \geq e^{1-\rho \bar{\nu}} \ln \frac{e}{e^{1-\rho \bar{\nu}}},
$$

respectively. Since function $a \ln \frac{e}{a}$ strictly increases on the segment $[0,1]$ and strictly decreases on the segment $[1, \infty)$, the previous inequalities are equivalent to the following ones

$$
e^{1-\rho \underline{\nu}} \geq \widetilde{a_{2}}, \quad e^{1-\rho \bar{\nu}} \leqslant \widetilde{a_{1}},
$$

where $\widetilde{a_{1}}, \widetilde{a_{2}}$ are roots to the equation $a \ln \frac{e}{a}=\frac{\widetilde{\Delta}}{\bar{\Delta}^{*}}, 0 \leqslant \widetilde{a_{1}} \leqslant 1 \leqslant \widetilde{a_{2}} \leqslant e$. Finding the logarithm of (30), we arrive at the relations

$$
\rho \underline{\underline{u}} \widetilde{a_{2}} \leqslant \widetilde{a_{2}} \ln \frac{e}{\widetilde{a_{2}}}=\frac{\widetilde{\Delta}}{\bar{\Delta}^{*}} \text { and } \rho \bar{\nu} \widetilde{a_{1}} \geq \widetilde{a_{1}} \ln \frac{e}{\widetilde{a_{1}}}=\frac{\widetilde{\Delta}}{\bar{\Delta}^{*}} .
$$


Hence, inequalities (21) and (22) of Theorem 2 can be shortly written in the equivalent form

$$
\rho \underline{\nu} \widetilde{a_{2}} \leqslant \frac{\widetilde{\Delta}}{\bar{\Delta}^{*}} \leqslant \rho \bar{\nu} \widetilde{a_{1}} .
$$

Proceeding in the same way, we write $(23)$ and $(24)$ as

$$
\frac{\widetilde{\Delta}}{\bar{\Delta}^{*}} \geq l^{-\rho} \ln \frac{e}{l^{-\rho}} \text { and } \frac{\widetilde{\Delta}}{\bar{\Delta}^{*}} \geq l^{\rho} \ln \frac{e}{l^{\rho}}
$$

that is equivalent to

$$
\widetilde{a_{1}} \geq l^{-\rho}, \quad \widetilde{a_{2}} \leqslant l^{\rho}
$$

Let us complete now inequality (21) by a lower estimate in terms of lower averaged and relative densities.

Proposition 6. The inequalities

$$
\underline{\Delta}^{*} \frac{e^{\rho \underline{\nu}-1}}{\rho \underline{\nu}} \leqslant \bar{\Delta}^{*} \leqslant \widetilde{\Delta} \frac{e^{\rho \underline{\nu}-1}}{\rho \underline{\nu}}
$$

hold true.

Proof. We need to justify only the left hand side of estimate (33) in a nontrivial case when $0<$ $\underline{\Delta}^{*} \leqslant \bar{\Delta}^{*}<+\infty$. We argue by contradiction assuming that $\bar{\Delta}^{*}<\underline{\Delta}^{*} \frac{e^{\rho \underline{\nu}-1}}{\rho \underline{\nu}}$. Then $\rho \underline{\nu} e^{1-\rho \underline{\nu}}<$ $\frac{\underline{\Delta}^{*}}{\overline{\bar{\Delta}}^{*}}$, or, which is equivalent, $e^{1-\rho \underline{\nu}} \ln \frac{e}{e^{1-\rho \underline{\nu}}}<\frac{{\underline{\Delta^{*}}}^{*}}{\bar{\Delta}^{*}}$. Therefore, $e^{1-\rho \underline{\nu}}>a_{2}$, where, as above, $a_{2}$ indicates the greater root of the equation $a \ln \frac{e}{a}=\frac{\triangleq^{*}}{\bar{\Delta}^{*}}$. In view of the left hand side in (13) it yields that

$$
\frac{\underline{\Delta}^{*}}{\overline{\bar{\Delta}}^{*}}=a_{2} \ln \frac{e}{a_{2}}>a_{2} \rho \underline{\nu} \geq a_{2} \rho \frac{\Delta^{*}}{\bar{\Delta}} \text {. }
$$

The obtained inequality $\frac{\Delta^{*}}{\overline{\bar{\Delta}}^{*}}>a_{2} \rho \frac{\Delta^{*}}{\bar{\Delta}}$ implies the estimate $\bar{\Delta}>a_{2} \rho \bar{\Delta}^{*}$ contradicting the latter relation in (2). The proof is complete.

We note once again in the case of a discrete measurable sequence determined by the condition $\underline{\Delta}^{*}=\widetilde{\Delta}$ the inequalities in Proposition 6 becomes identities justifying the exactness of estimates $(33)$.

In work [11], for the roots $a_{1}$ and $a_{2}$ of equation $a \ln \frac{e}{a}=\frac{\Delta^{*}}{\bar{\Delta}^{*}}$ the representation

$$
a_{1}=e q^{\frac{q}{1-q}}, \quad a_{2}=e q^{\frac{1}{1-q}}
$$

was found via the parameter $q=\frac{a_{2}}{a_{1}}$. It was shown that in the case of a discrete measurable sequence of complex numbers with the lacunarity index $l$ the identity $q=l^{\rho}$ holds true. We employ these facts to obtain the next result; it is interesting to compare it with estimates (23), (24) in Theorem 2.

Proposition 7. For an arbitrary sequence of complex numbers with finite positive averaged densities $\underline{\Delta}^{*}, \bar{\Delta}^{*}$ and the lacunarity index $l$ the inequalities

$$
\begin{gathered}
\bar{\Delta}^{*} \geq \underline{\Delta}^{*} l^{\frac{\rho}{l^{\rho}-1}} \frac{l^{\rho}-1}{e \ln l^{\rho}}, \\
\Delta^{*} \frac{l^{\rho}-1}{a_{2} \ln l^{\rho}} \leqslant \bar{\Delta}^{*} \leqslant \Delta^{*} \frac{l^{\rho}-1}{a_{1} l^{\rho} \ln l^{\rho}} .
\end{gathered}
$$


As above, $a_{1}$ and $a_{2}$ are the roots to equation $a \ln \frac{e}{a}=\frac{\Delta^{*}}{\overline{\bar{\Delta}}^{*}}$. The presence of the discrete measurability provides the identities in (34), (35).

Proof. It follows from inequalities (5) and (1) that $l^{\rho} \leqslant \frac{\bar{\Delta}}{\Delta} \leqslant \frac{a_{2}}{a_{1}}=q$, i.e., $l^{\rho} \leqslant q$. Employing Item I of Lemma, we see that the expression $a_{2}(q)=e q^{\frac{1}{1-q}}$ increases as $q$ does. Hence, $a_{2}(q) \geq a_{2}\left(l^{\rho}\right)$. Since the function $a \ln \frac{e}{a}$ decreases on the interval $[1,+\infty)$, we have

$$
\frac{{\underline{\Delta^{*}}}^{*}}{\bar{\Delta}^{*}}=a_{2}(q) \ln \frac{e}{a_{2}(q)} \leqslant a_{2}\left(l^{\rho}\right) \ln \frac{e}{a_{2}\left(l^{\rho}\right)}=e^{\frac{\rho}{1-l^{\rho}}} \frac{\ln l^{\rho}}{l^{\rho}-1}
$$

that implies (34). Inequalities (35) can be proven by similar arguments if one also notes that $a_{1}=a_{1}(q)=e q^{\frac{q}{1-q}}$ decreases as $q$ increases. For instance, the right hand side of (35) follows from the relations

The proof is complete.

$$
\frac{\underline{\Delta}^{*}}{\overline{\bar{\Delta}}^{*}}=a_{1}(q) \ln \frac{e}{a_{1}(q)} \geq a_{1} \ln \frac{e}{a_{1}\left(l^{\rho}\right)}=a_{1} l^{\rho} \frac{\ln l^{\rho}}{l^{\rho}-1} .
$$

As it has been observed, the discrete measurability of a sequence does not imply its measurability. It is interesting to know what are the additional conditions ensuring the measurability. As we see by Proposition 1, one of such condition is a weak lacunarity of a sequence. Indeed, as $l=1$, it follows from (6) that $\underline{\Delta}^{*} \leqslant \bar{\Delta}^{*} \leqslant \widetilde{\Delta}=\underline{\Delta}^{*}$, i.e., $\underline{\Delta}^{*}=\bar{\Delta}^{*}$. Another condition is the internal measurability of a sequence that implies, in accordance with inequality (8) in Proposition 1, its weak lacunarity. However, we can obtain a stronger statement from Theorem 2.

Proposition 8. Let $\Lambda$ be a discrete measurable sequence of complex numbers. Then each of the conditions $\underline{\nu}=\frac{1}{\rho}$ or $\bar{\nu}=\frac{1}{\rho}$ implies the measurability of this sequence.

Proof. The discrete measurability means that $\widetilde{\Delta}=\Delta^{*}$. And each of the conditions from the proposition, in accordance with formulae (21) and (22), imply the inequalities $\underline{\Delta}^{*} \leqslant \bar{\Delta}^{*} \leqslant \widetilde{\Delta} \leqslant$ $\Delta^{*}$ that yields the measurability.

There appears a natural question: whether only the weak lacunarity or only the internal measurability (with the discrete one) of a sequence implies its measurability? We shall give the negative answer to both the questions by using the notion of Valiron's specified ordering. We remind that it indicates a differentiable on $\mathbb{R}_{+}$function $\rho(x)$ satisfying two conditions $\lim _{x \rightarrow+\infty} \rho(x)=\rho>0$ and $\lim _{x \rightarrow+\infty} \rho^{\prime}(x) x \ln x=0$ which are equivalent to one condition $\lim _{x \rightarrow+\infty} \frac{x h^{\prime}(x)}{h(x)}=\rho$ for the function $h(x)=x^{\rho(x)}$ or, which is more convenient for our aims, to the condition

for the function $L(x)=h(x) x^{-\rho}$.

$$
\lim _{x \rightarrow+\infty} \frac{x L^{\prime}(x)}{L(x)}=0
$$

Proposition 9. Given any $\rho>0, \beta>0$ and $\alpha \in[0, \beta]$, there exist internal measurable weakly lacunar sequences with $\rho$-densities $\triangleq=\alpha$ and $\bar{\Delta}=\beta$.

Proof. Suppose numbers $\rho, \beta$ and $\alpha$ satisfy the hypothesis of the proposition. If $\alpha>0$, to construct the desired sequence we take the function $\alpha(x) \equiv \alpha$. If $\alpha=0$, we let $\alpha(x)=\ln ^{-\gamma} x$, where $\gamma \in(0,1)$. As $L(x)$ we choose then the function

$$
L(x)=\frac{1}{\rho} \sqrt{\alpha(x) \beta}\left(\sqrt{\frac{\beta}{\alpha(x)}}\right)^{\sin \ln ^{\gamma} x},
$$


and it is easy to check that it satisfies condition (36). We define then the function $n(x)=$ $\left[x h^{\prime}(x)\right]$ and choose sequence $\Lambda$ so that its counting function coincides with $n(x)$. Here $h(x)=$ $x^{\rho} L(x)$, and $[x]$ denotes the integer part of number $x$. The results of monograph [7] follow that as $x \rightarrow+\infty$, conditions $N(x) \sim h(x)$ and $n(x) \sim x h^{\prime}(x)$ for the counting functions of $\Lambda$ are equivalent. Sequence $\Lambda$ is internal measurable since by the construction it satisfies

$$
\lim _{x \rightarrow+\infty} \frac{N(x)}{n(x)}=\lim _{x \rightarrow+\infty} \frac{h(x)}{x h^{\prime}(x)}=\frac{1}{\rho} .
$$

On the other hand,

$$
\begin{aligned}
\rho \underline{\Delta}^{*} & =\rho \underline{\lim }_{x \rightarrow+\infty} \frac{N(x)}{x^{\rho}}=\rho \underline{\lim }_{x \rightarrow+\infty} \frac{h(x)}{x^{\rho}}=\rho \underline{\lim }_{x \rightarrow+\infty} L(x) \\
& =\varliminf_{x \rightarrow+\infty} \sqrt{\alpha(x) \beta}\left(\sqrt{\frac{\beta}{\alpha(x)}}\right)^{\operatorname{lin}^{\gamma} \ln ^{\gamma} x}=\underline{\lim }_{x \rightarrow+\infty} \sqrt{\alpha^{2}(x)}=\lim _{x \rightarrow+\infty} \alpha(x)=\alpha
\end{aligned}
$$

and

$$
\rho \bar{\Delta}^{*}=\rho \varlimsup_{x \rightarrow+\infty} \frac{N(x)}{x^{\rho}}=\rho \varlimsup_{x \rightarrow+\infty} L(x)=\varlimsup_{x \rightarrow+\infty} \sqrt{\alpha(x) \beta}\left(\sqrt{\frac{\beta}{\alpha(x)}}\right)^{\sin ^{\gamma} \ln ^{\gamma}}=\beta .
$$

Applying the corollary of Proposition 2, we obtain

$$
\underline{\Delta}=\rho \underline{\Delta}^{*}=\alpha \text { and } \bar{\Delta}=\rho \bar{\Delta}^{*}=\beta .
$$

Thus, the constructed sequence has prescribed $\rho$-densities, is internal measurable and its weak lacunarity follows from inequality (8) of Proposition 1. The proof is complete.

Summarizing the above arguments, we can formulate the following statement.

Proposition 10. An arbitrary sequence $\Lambda=\left\{\lambda_{n}\right\}_{n=1}^{\infty}$ of complex numbers with the convergence index $\rho>0$ and $0<\bar{\Delta}<\infty$ is measurable if and only if it is discrete measurable and at least one of the following conditions holds

a) sequence $\Lambda$ is weakly lacunar, i.e., $l_{\Lambda}=\varlimsup_{n \rightarrow \infty} \frac{\left|\lambda_{n+1}\right|}{\left|\lambda_{n}\right|}=1$;

b) the condition $\underline{\nu}=\varliminf_{x \rightarrow+\infty} \frac{N(x)}{n(x)}=\frac{1}{\rho}$ holds true;

c) the condition $\bar{\nu}=\varlimsup_{x \rightarrow+\infty} \frac{N(x)}{n(x)}=\frac{1}{\rho}$ holds true;

d) sequence $\Lambda$ is internal measurable, i.e., there exists the limit

$$
\lim _{x \rightarrow+\infty} \frac{N(x)}{n(x)}\left(=\frac{1}{\rho}\right)
$$

Proof. The sufficiency of the discrete measurability of sequence $\Lambda$ with each of conditions a) or d) for its measurability has already been discussed before Proposition 8, and similar discussion for conditions b) and c) was made in Proposition 8.

Suppose now sequence $\Lambda$ is measurable, i.e., $\Delta=\bar{\Delta}=\Delta$. Its discrete measurability follows immediately from the definition. The weak lacunarity follows from inequality (5) of Proposition 1, and its internal measurability is implied by inequalities (1) and the existence of the limit

$$
\nu=\lim _{x \rightarrow+\infty} \frac{N(x)}{n(x)}=\lim _{x \rightarrow+\infty} \frac{N(x) / x^{\rho}}{n(x) / x^{\rho}}=\frac{\Delta / \rho}{\Delta}=\frac{1}{\rho} .
$$

The proof is complete. 


\section{BIBLIOGRAPHY}

1. B.Ya. Levin. Distribution of zeros of entire functions. GITTL, Moscow, 1956. [Amer. Math. Soc., Providence RI, 1980.]

2. A.F. Leont'ev. Exponentials series. Nauka, Moscow. 1976. (in Russian).

3. R.P. Boas. Entire functions. Academic Press, New-York, 1954.

4. A.A. Gol'dberg. The integral over a semi-additive measure and its application to the theory of entire functions. IV // Matem. Sbornik. 1965. V. 66, No. 3. P. 411-457. (in Russian.)

5. A.A. Kondratyk. On extremal indicator of entire functions with positive zeroes // Sibir. Matem. Zhurn. 1970. V. 11, No. 5. P. 1084-1092. (in Russian).

6. B.N. Khabibullin. Completeness of exponential series and the uniqueness sets. 4ed. Bashkir State Univeristy, Ufa, 2012. (in Russian.)

7. G.G. Braichev. An introduction to the theory of growth of convex and entire functions, Prometej, Moscow, 2005. (in Russian.)

8. G.G. Braichev. The exact bounds of the types of entire functions of order less than unity with the zeros located on the ray // Ufimskij Matem. Zhurn. 2012. V. 4, No. 1. P. 29-37. [Ufa Math. J. 2012. V. 4, No. 1. P. 27-34.]

9. G.G. Braichev. The least type of an entire function of order $\rho \in(0,1)$ having positive zeros with prescribed averaged densities // Matem. Sbornik. 2012. V. 203, No. 7. P. 31-56. [Sb. Math. 2012. V. 203, No. 7. P. 950-975.]

10. G.G. Braichev, V.B. Sherstyukov. On the growth of entire functions with discretely measurable zeros // Matem. Zametki. 2012. V. 91, No. 5. P. 674-690. [Math. Notes. 2012. V. 91, No. 5-6. P. 630-644.]

11. G.G. Braichev, V.B. Sherstyukov. Exact relatins between zeroes densities for entire function of finite order // Matematychni Studii. 2008. V. 30, No. 2. P. 183-188. (in Russian.)

12. G. Pólya and G. Szegö. Problems and Theorems in Analysis II: Theory of Functions. Zeros. Polynomials. Determinants. Number Theory. Geometry. Springer, Berlin. 1976.

Georgii Genrikhovich Braichev

Moscow State Pedagogical University,

M. Pirogovskaya str., 1, 199296, Moscow, Russia

E-mail: braichev@mail.ru 